\title{
THICK FILM TEMPERATURE SENSORS USING STANDARD PASTES
}

\author{
I. JANOSKA and M.R. HASKARD \\ South Australian Institute of Technology, The Levels, Pooraka, P.O. Box 1, Ingle Farm 5098, South Australia
}

Standard thick film resistor pastes exhibit changes in their electrical characteristics when printed on top of dielectric layers. Of particular interest is the inherent change in their temperature coefficient of resistance. Simple temperature sensors were formed by deliberately printing thick film resistor pastes on top of larger area dielectric layers. Temperature tests carried out on these devices have shown that by selecting the correct paste combination and resistor aspect ratio stable, repeatable, temperature sensors with good linearity can be manufactured. A comparison is made of these sensors to other commercially available products currently used in the thick film industry.

\section{INTRODUCTION}

A wide range of components exists for use as sensors in thick film systems. They range from packaged chips, which may be mounted or bonded to the substrate material, through to special temperature sensitive pastes specifically designed with a high temperature coefficient of resistance (TCR). Each has its own particular class of advantages and disadvantages, and the choice of which to use is dependent on the application, cost and many other factors. All are special components and there would be an obvious advantage if temperature sensors could be made by using a manufacturer's range of standard pastes.

It is well known that resistor pastes display piezo resistive effects. ${ }^{1}$ Normally this is a disadvantage. However, if such a paste is printed on to an insulating pastes with a different temperature expansion coefficient, then the resulting combination gives rise to a temperature sensor with improved sensitivity. Such sensors are produced using the same pastes as used for other passive components in the circuit and therefore need no special processing steps.

The properties required of a temperature sensor depend somewhat on the application. For an on/off threshold type of circuit, even a non-linear sensor is satisfactory providing its sensitivity is adequate. On the other hand, to measure temperature accurately over a wide dynamic range a sensor with greater linearity is required. Some necessary requirements of this type of device are as follows. It should be stable, well characterised with a tight manufacturing tolerance having highly linear characteristics and showing no hysteresis effects.

In investigating the feasibility of these sensors due consideration was given to these properties. A comparison was also made with a number of other temperature sensors that are commercially available for use in thick film circuits.

\section{CONSTRUCTION OF STANDARD PASTES SENSORS}

The manufacturing process and construction of these sensors is straightforward (refer Figure 1). First a layer of dielectric paste is printed on to the substrate material at the location of the sensor. It is not necessary to remove pin holes in the dielectric with a second over-printing. The dielectric is then baked and fired. Conductor tracks are then printed over each end of the dielectric to terminate the final resistor layer. The conductor is also baked and tired, after which the resistor layer is printed and fired according to its profile. Note that the dielectric layer underneath is larger than the 

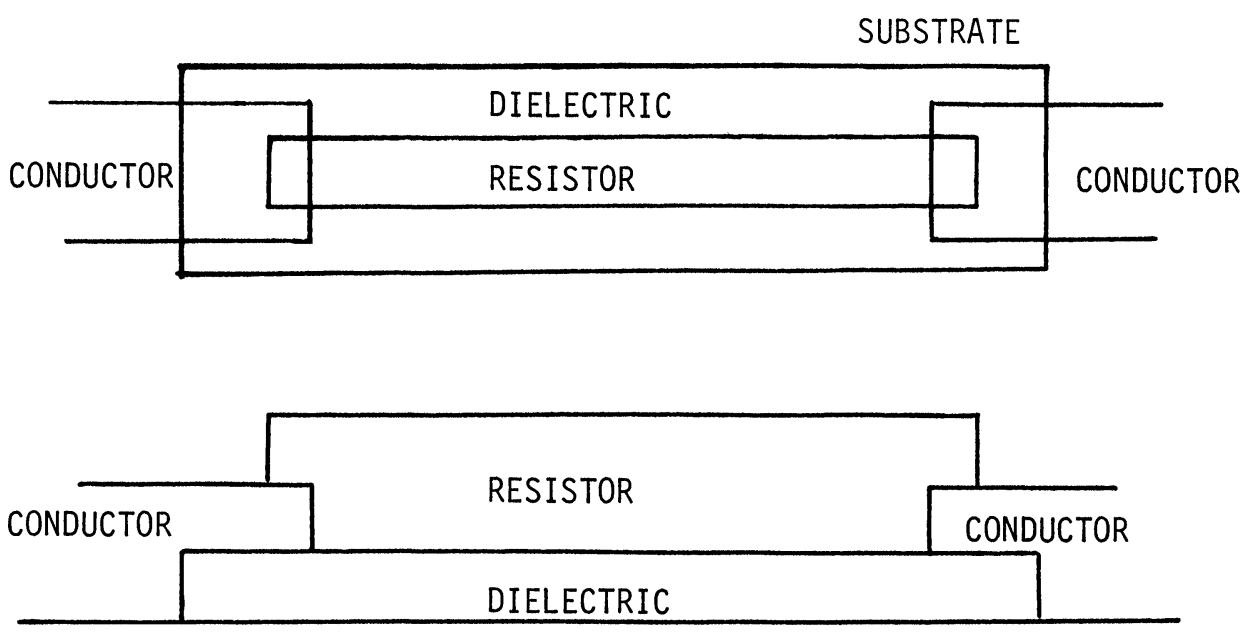

SUBSTRATE

FIGURE 1 Construction of incompatible paste temperature sensor.

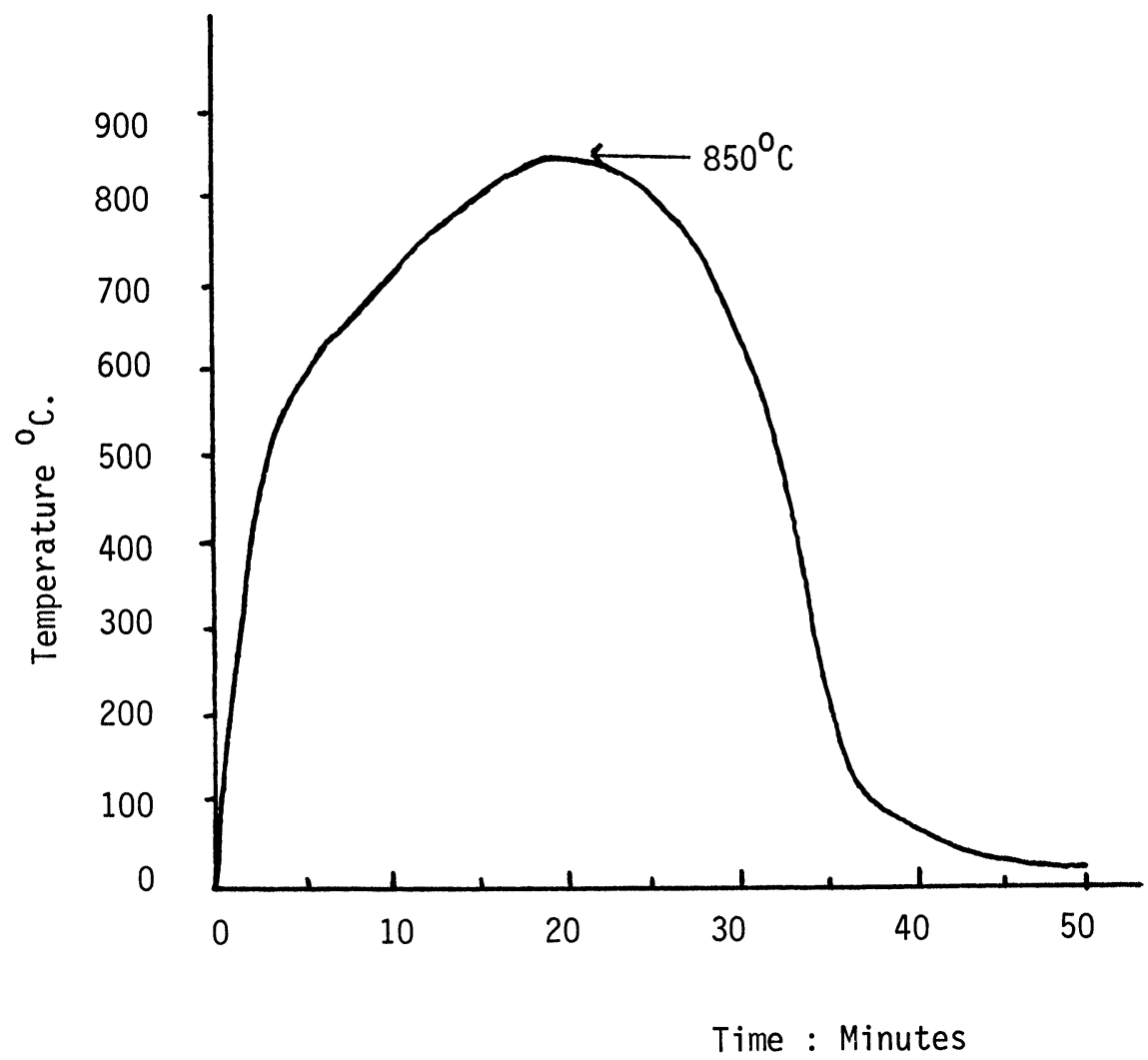

FIGURE 2 Time-temperature profile for furnace employed to produce the sensors. 
TABLE I

Pastes investigated to manufacture incompatible paste temperature sensors.

\begin{tabular}{llll}
\hline Substrate & Dielectric & Resistor & Conductor \\
\hline A1-4 & Du Pont 9841 & ESL 2813 & ESL 9635 \\
B1-4 & Du Pont 9841 & ESL 2814 & ESL 9635 \\
C1-4 & ESL 5608 & ESL 2813 & ESL 9635 \\
D1-4 & ESL 4608 & ESL 2814 & ESL 9635 \\
E1-4 & ESL 45125 & ESL 2813 & ESL 9635 \\
F1-4 & ESL 45125 & ESL 2814 & ESL 9635 \\
\hline
\end{tabular}

ESL 2813 is a $1 \mathrm{k} \mathrm{ohm} /$ square paste and ESL 2814 is a $10 \mathrm{k} \mathrm{ohm} /$ square paste.

resistors on top, to ensure that no part of the resistor comes into contact with the substrate material. On the test substrates, for each sensor produced in this way an identical resistor, without a dielectric layer, was printed alongside the sensor so that changes in characteristics between the two devices could be easily compared. For convenience, resistors printed on dielectric layers are abbreviated to RDIE. Similarly, those printed without a dielectric layer are abbreviated to RSUB.

\section{STANDARD PASTE SENSORS}

The sensors under investigation consisted of standard resistor and dielectric pastes made by ESL and Du Pont. After printing all pastes were allowed to settle for 8 minutes, dried at $125^{\circ} \mathrm{C}$ for 10 minutes and then fired using the time temperature profile shown in Figure 2. The substrate material was Coors 96\% alumina type ADS-96F, of dimensions $25 \mathrm{~mm} \times 50 \mathrm{~mm} \times 0.635 \mathrm{~mm}$. The 24 substrates that were manufactured and tested were broken into six groups, labelled A to F, each of four substrates numbered 1 to 4. Table I summarises the combinations of pastes used in each group.

Because of the large number of resistor/dielectric combinations and types of tests that were carried out, the substrate design needed to be multifunctional, incorporating as many different sensors as possible. Each substrate contained 24 resistors in RSUB and RDIE pairs, 6 of these pairs were printed longitudinally (parallel to the squeegee) and 6 pairs printed transversely (perpendicular to the squeegee). A general crosssection of aspect ratios was chosen. These were $10,5,3,1,1 / 3$ and $1 / 5$ which were similar to those used by K.E.G. Pitt and R.J. Gledhill. ${ }^{2}$ The two printing directions were required, firstly to observe the effects of printing direction and, secondly, to examine the effects of two-directional strain under an applied planar force. Figure 3 is a photo of a test subtrate. The substrates had $3.5 \mathrm{~mm}$ of bare alumina at each end; used to grip the substrate during the strain tests.

During the temperature testing each resistor was measured at seven temperatures, namely $20,40,60,80,100,120$ and 20 degrees Celsius. Note that two measurements were made at 20 degrees, one at the beginning and one at the end so as to assess the possibility of distortion or ageing due to the thermal extremes of the test. Some 8736 measurements were recorded using a Systron Donner $51 / 2$ digit resistance meter.

Each resistor was individually labelled using a code to classify the physical parameters of the resistor. For instance the unique resistor, A3RDL5, is located in group $\mathrm{A}$, on substrate 3, it is a resistor on a dielectric layer (RDIE), which is longitudinally printed with an aspect ratio of 5. A six dimensional array was used to store all of the results during computer processing using the above conditions or 


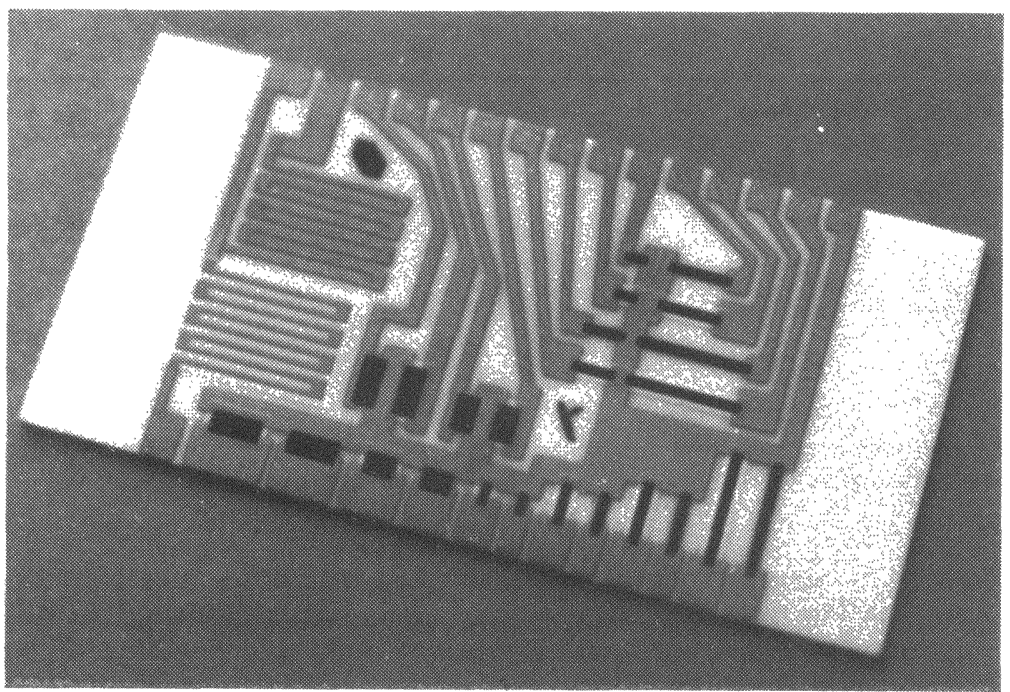

FIGURE 3 Sensor test substrate (Blocks are for applying axial stain).

physical parameters as the first five dimensions, with the sixth dimension corresponding to the temperature at which the measurement was taken. The program computed mean values, percentage deviations and slope or TCR calculations on each set of points. It also provided different measures of linearity using various figures of merit. These results for each resistor were compared and sorted into various groups, the results being printed in a format that allowed rapid inspection to extract both trends and desirable combinations.

\section{RESULTS PROCESSING AND ANALYSIS}

Much of the processed data was in ratio form. These ratios gave a simple method of comparison between RDIE and RSUB pair characteristics. Possibly one of the most obvious and consistent results was the increase in absolute value of resistance for RDIE resistors compared to RSUB types. This increase was primarily dependent on the type of dielectric paste used, more so than the resistor paste or aspect ratio. This tends to suggest that the increase is due to the chemical nature of the dielectric paste as the process would hold all of the physical parameters, such as printing thickness, approximately constant. A close investigation of the pastes rheology may provide some insight into which of the primary components or additives brings about this phenomenon. It was not the purpose of the investigation to determine the process by which the dielectric paste affects the resistor's characteristics, but merely to determine the usefulness of these characteristic changes from a designer's point of view. These increases in absolute value were averaged and plotted in Figures 4a and b. The graph shows the ratio RDIE/RSUB, taken at $20^{\circ} \mathrm{C}$., versus aspect ratio for each of the six groups. These are only for the transversely printed resistors, as the result for the longitudinal device was almost identical in shape but generally $25 \%$ lower in magnitude. Groups A, B, C and D were found to have a near constant RDIE/RSUB ratio for all aspect ratios. The results varied from 1.2 to 2.5 within these four groups. Figure 4a shows that the increase from RSUB to RDIE is strongly affected by the type of resistor paste and to a lesser extent the dielectric paste. 


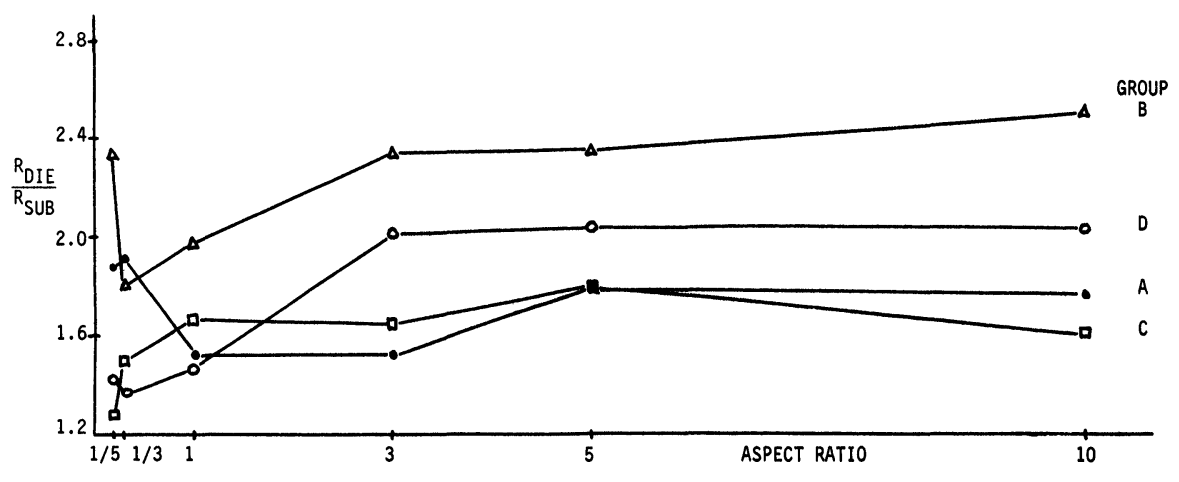

(a)

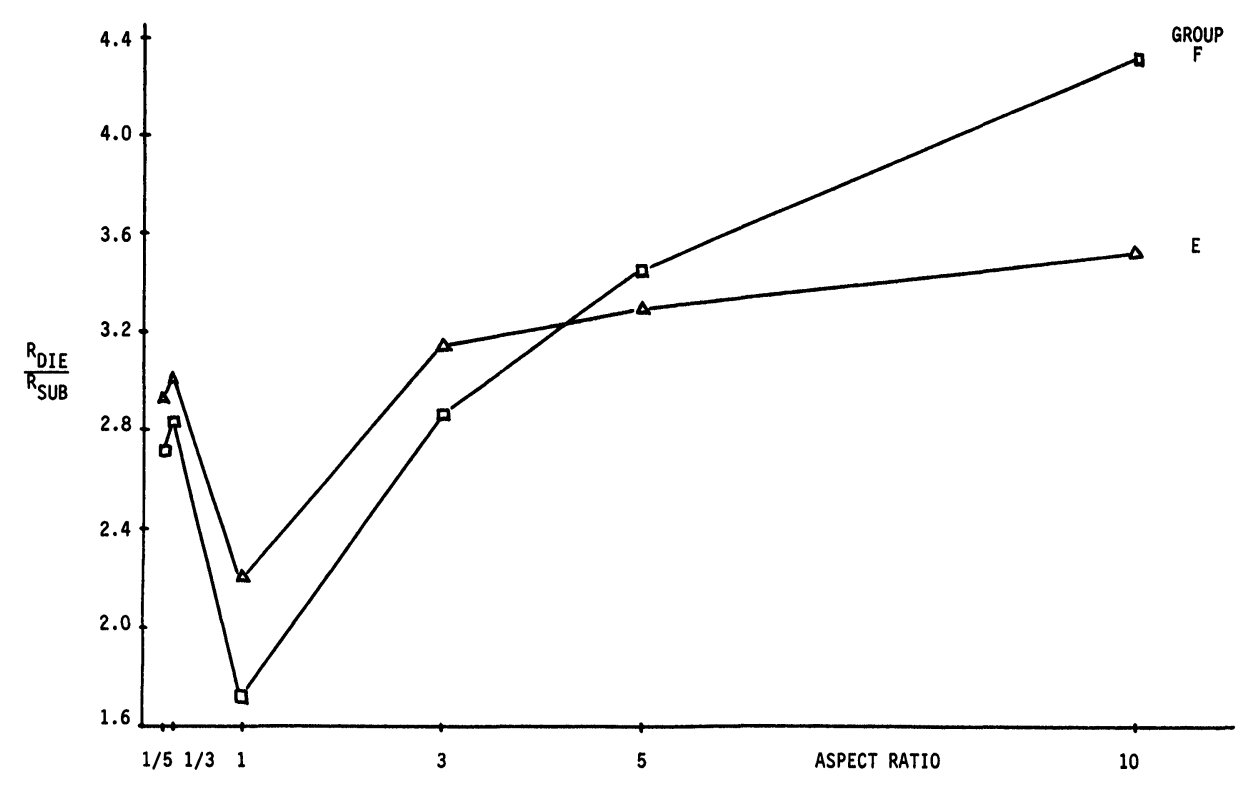

(b)

FIGURE 4 Ratio of resistance on dielectric to resistance on substrate versus aspect ratio for various paste combinations.

Group E and F substrates (Figure 4b) incorporating the ESL 45125 dielectric, showed the largest increase of up to 4.6 times. Group F was the worst behaved as the curve was not as constant with aspect ratio but showed a consistent linear increase. Another consistent feature of both these groups was the valley point found at an aspect ratio of 1 . A possible explanation of these shapes is as follows. Firstly it should be remembered that large aspect ratio resistors on dielectric are predominantly a three layer system consisting of substrate, dielectric and resistor pastes. Fo' small aspect ratios the conductor paste must be included and the device increases to four layers. Ignoring the conductor for the present and considering a square resistor some changes 


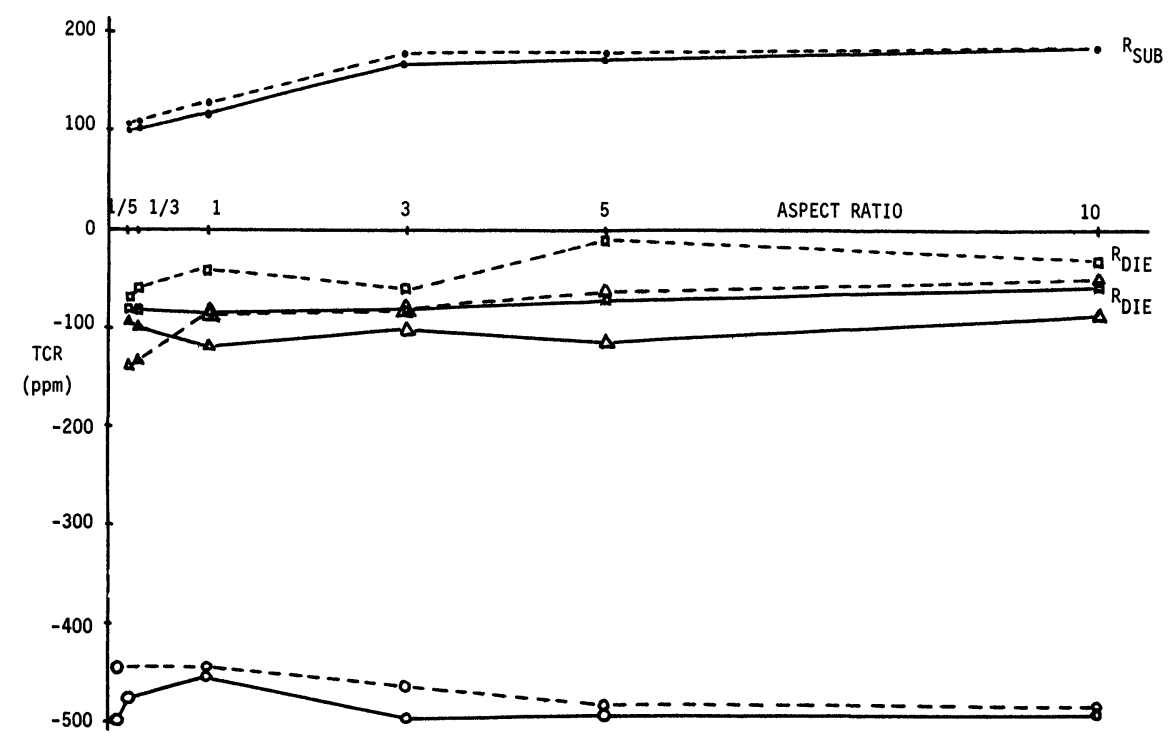

FIGURE 5 Temperature coefficient of resistance versus aspect ratio for group $A(\triangle), C(\square)$ and $E(O)$ for $1 \mathrm{kohm} / \mathrm{square}$ resistance paste. Solid lines are for transverse and dotted lines for longitudinal sensors.

in resistance due to chemical and mechanical reasons will be similar in both the $\mathrm{X}$ and $Y$ directions so that their influence on the resultant sheet resistance will cancel. Adding in the conductor will bring about an out of balance of these $\mathrm{X}$ and $\mathrm{Y}$ resistance changes. However, the overall change will be minimal when compared to other aspect ratio resistors.

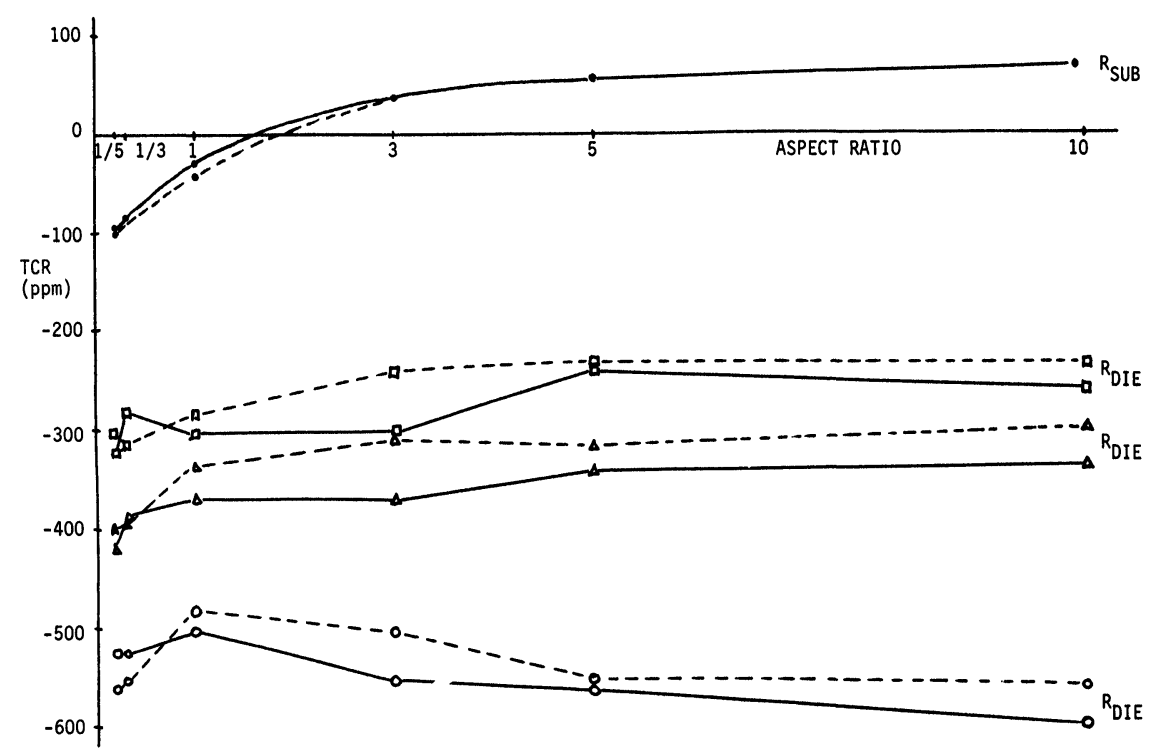

FIGURE 6 Temperature coefficient of resistance versus aspect ratio for group $B(\triangle), D(\square)$ and $F(O)$ for $10 \mathrm{kohm} /$ square resistor paste. Solid lines are for transverse and dotted line longitudinal sensors. 
As mentioned earlier, each group had four identical substrates, numbered 1 to 4 . A resistor was thus reproduced 4 times, making it possible to calculate a percentage spread over these 4 resistors. The tolerance was calculated as the \% spread around the mean value. It is interesting to note that this tolerance was near identical for RSUB and RDIE resistors irrespective of paste combination. This shows that the resistor to resistor tolerances, within a batch, remains virtually unchanged for resistors on dielectric compared to resistors printed on substrate. To some extent it is irrelevant as to what the absolute value tolerances were, as this is primarily dependent on the quality of the pastes and the process. It is more meaningful to note that RSUB and RDIE sensors seem to have the same tolerances within a batch. Tolerances calculated varied from $1.0 \%$ to $20.0 \%$ over the 24 substrates produced, being independent of paste combination, aspect ratio and printing direction.

Following this the program processed the temperature dependent characteristics of the RSUB and RDIE sensors. Several observations were made relating to the temperature coefficient of resistance, as well as the linearity of their temperature curves. In both cases comparisons were made between the RSUB and RDIE sensors. The resulting characteristic TCR versus aspect ratio, for all groups, is shown in Figures 5 and 6. Firstly, from a general observation of all six groups, it can be seen that the curves for the RSUB sensors are more well behaved over the whole range of aspect ratios. In fact both transverse and longitudinal resistors behave identically with aspect ratio. With RDIE sensors for aspect ratios less than 3, there are some variations; however above 3 the TCR's become almost constant, a useful property when designing sensors.

For groups A and C, the ratio of RDIE TCR's to RSUB TCR's range typically from 0.3 to 1.0 throughout both groups. This suggests an increase in temperature stability for RDIE sensors, which is a desirable property. On the other hand groups B, D, E and F show a general increase in the absolute value of TCR's. This increase is dependent on the combination of pastes used, and to a lesser extent the aspect ratio employed. Again the best results were found with groups $E$ and $F$ which incorporated the 45125 dielectric paste. Group B also showed promise. (It is interesting to note once more that these results suggest a large dependence on dielectric paste type and a small dependence on resistor paste). The general increase in TCR of the RDIE sensors in groups B, D, E and F ranges from 5.7 to 10 times. Table 2 summarises the relative increase of RDIE TCR's compared to RSUB TCR's for sensors with an aspect ratio of 5 and printed in a longitudinal direction. The average TCR(PPM) of RDIE sensors in each group is also provided in the table.

Another interesting result was the relative change in sign of TCR (slope) between RSUB and RDIE sensors which can be seen in Figures 5 and 6 . Notice that the TCR for all of the manufactured RDIE sensors were negative in sign whereas RSUB types

TABLE II

Temperature coefficient of resistance ratios and values for sensors of aspect ratio of 5 and printed longitudinally.

\begin{tabular}{lll}
\hline Group & {$[$ RDIE TCR $] /[$ RSUB TCR $]$} & Approx. TCR (ppm) \\
\hline A & 0.4 & -70 \\
B & 5.7 & -310 \\
C & 0.06 & -10 \\
D & 4.0 & -220 \\
E & 2.5 & -450 \\
F & 10.0 & -550 \\
\hline
\end{tabular}




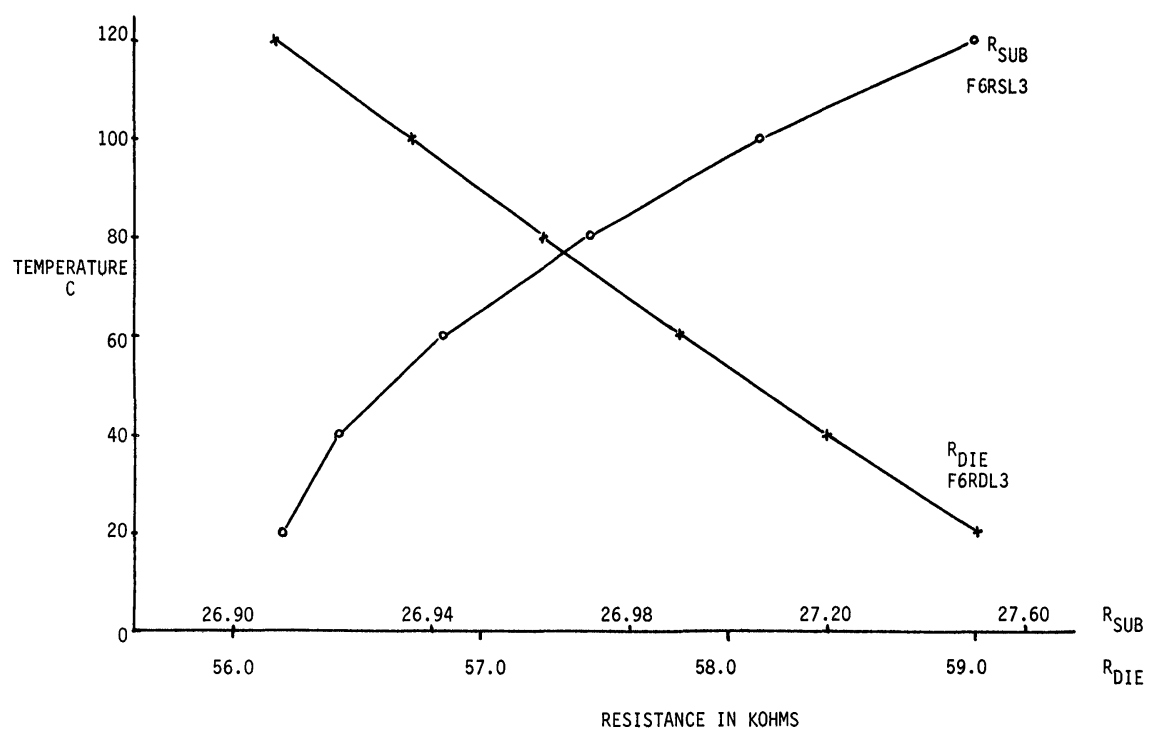

FIGURE 7 Typical resistance versus temperature curve for a resistance on substrate and on dielectric material.

normally had positive TCR's for aspect ratio greater than 1 . This change of sign is also very apparent in Figure 7 showing plots of typical resistance versus temperature curves. curves.

Possibly the most remarkable result found throughout the whole investigation and illustrated in Figure 7 was the obvious improvement in linearity of RDIE sensors compared to the RSUB types. This general increase in linearity was most apparent with the $10 \mathrm{k} \mathrm{ohm}$ paste but less so with the $1 \mathrm{k} \mathrm{ohm}$ paste, showing that this paramter is largely dependent on the type of resistor paste in use. For the $1 \mathrm{k} \mathrm{ohm} \mathrm{pastes,} \mathrm{linearity}$ ratios (ratio of sum of the square of residuals from a line of best fit, that is RDIE residuals/RSUB residuals) varied from 0.5 to 8.0 , implying a decrease in linearity in some cases. Generally the best results occurred in the E group substrates, with the worst being those in the $\mathrm{C}$ group. For the $10 \mathrm{k} \mathrm{ohm}$ paste the results were much improved. The B group gave ratios ranging from 0.2 to 0.5 , and similarly, ratios of 0.3 to 0.5 were found with the $\mathrm{D}$ group. The $\mathrm{F}$ group showed ratios ranging from 0.1 to 0.5 , and the best of these results corresponded to sensors with aspect ratios of 1 and 3 .

Finally, a distortion figure was calculated by comparing values of the absolute resistance measured twice at the ambient temperature. The difference between the to $20^{\circ} \mathrm{C}$ values was an indication of the possible hysteresis that the sensor may have undergone throughout a thermal cycle. All differences were considerably less than $1 \%$ suggesting there is little hysteresis effect.

\section{COMPARISON WITH OTHER SENSORS}

The performances of the standard paste sensors were firstly compared with a special temperature sensitive paste typical of that used in present day thick film technology. The special paste was the ESL NTC 2414 , specified as a $10 \mathrm{k} \mathrm{ohm} / \mathrm{square}$ paste at $25^{\circ} \mathrm{C}$. It comes from a family having various sheet resistivities and has a typical TCR of -7000 $\mathrm{ppm} / \mathrm{deg}$.C over the temperature range of 25 to $125^{\circ} \mathrm{C}$. This paste was tested and 


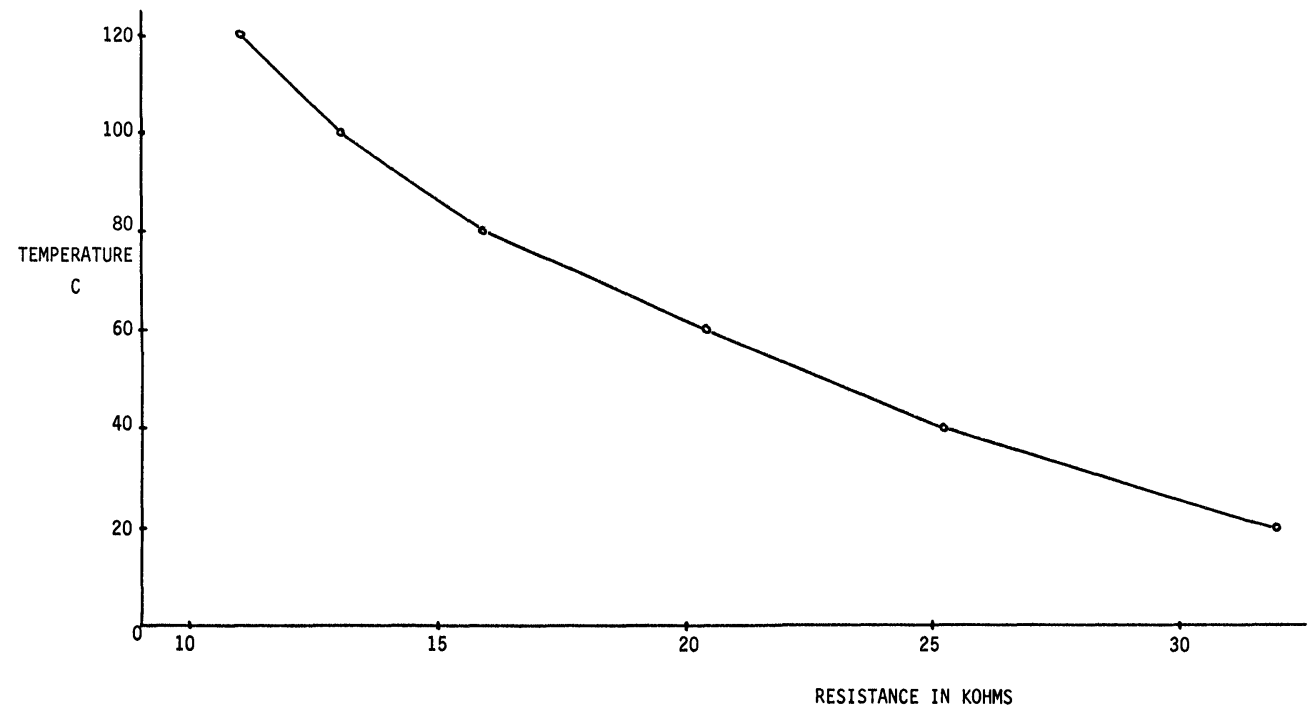

FIGURE 8 Typical resistance versus temperature curve for a special temperature sensitive paste type XSL3.

processed in an identical manner to the standard paste sensors using the identical screens from the previous substrates. For interest, this paste was also printed on top of the same dielectric layers providing the same RDIE and RSUB pairs as before.

The results suggest that there are no obvious disadvantages found in printing this special paste on top of a dielectric layer. Values of the TCR's ranged from -4500 to $-8000 \mathrm{ppm}$ depending on the dielectric paste. When compared to the RSUB sensors (at $25^{\circ} \mathrm{C}$ ) there was a noticeable increase in the absolute resistance value for RDIE devices; increases far greater than observed with the standard paste sensors. Unlike the temperature versus resistance curves for the standard paste sensors, the special paste curves showed no apparent change in linearity between the RDIE and RSUB types. All of these curves showed the same general shape as well as sign of TCR. The addition of the dielectric layer seemed only to provide a positive shift of the resistance versus temperature curve along the resistance axis. The only obvious advantage in using this property would be if extremely high impedance temperature sensitive devices were required in some special application. Figure 7 shows the relative shape of a typical special paste sensor curve. A visual comparison of linearity between Figures 7 and 8 tends to suggest that the RDIE incompatible paste sensors have a slightly better overall characteristic although they have a much lower TCR compared to the special paste sensors.

Two further temperature sensors were investigated briefly and their characteristics were compared with the incompatible paste type sensors. They were both taken from the Philips range of products. The first was a silicon planar plastic encapsulated device, KTY $81 \mathrm{~B} 1$, which is intended for use on printed circuit boards. They are nominally $1 \mathrm{k}$ $1 \% \mathrm{ohms}$ at $25^{\circ}$ with a TCR of $7500 \mathrm{ppm}$. The second was a PTC thermistor type 2322672 91031. These are small bead type devices which are intended for use in thick film circuits. The TCR is specified as being 18 to $38 \% /{ }^{\circ} \mathrm{C}$. The characteristics for both of these sensors are provided in Figure 9.

As the manufacturer's data sheet specifies, the KTY 81 B1 silicon planar sensor has a very linear characteristic with an extremely tight tolerance. These characteristics are no doubt useful for temperature measurement although the plastic encapsulation is 


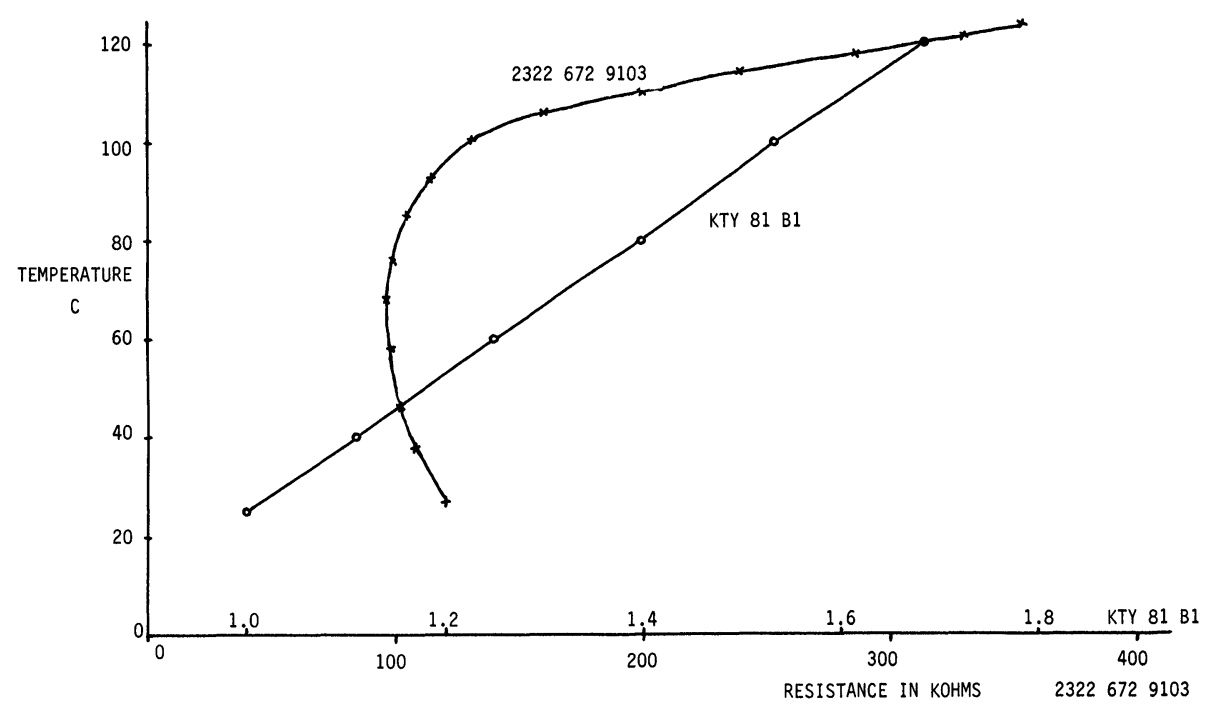

FIGURE 9 Typical resistance versus temperature characteristics for Philips sensors KTY 81 B1 (silicon) and 232267291031 (thermistor).

responsible for a relatively high thermal time constant giving it a slow response. The PTC thermistors showed poor linearity and a limited useful range.

\section{CONCLUSIONS}

From the analysis and comparison of the standard paste sensors it can be concluded that these devices appear to be a viable option when choosing temperature sensors for thick film applications. Their characteristics show good linearity although their temperature coefficients of resistance are relatively small compared with some of the technology presently in use. These sensors have several advantages over the special temperature sensitive pastes. Firstly, they can be constructed from standard resistor and dielectric pastes already in stock. They require no special production procedures and no bonding of devices to the substrate is necessary, as required with pre-manufactured chip sensors. Often the same screen used for printing other passive components can be employed. Furnace peak firing temperature and firing profile need not be adjusted as is often the case for some special paste sensors.

During the investigation three batches of standard paste sensors were manufactured to provide some indication of the batch repeatability. This test showed that the firing of the dielectric part is very critical if repeatable absolute values of sensor resistance and TCR are to be achieved. During the manufacture of the second batch a very prolonged time temperature profile was used, yet the TCR was still within $30 \%$.

The standard paste sensors have been used successfully in two applications giving accurate measurement and control of temperature. Resistor ratios or bridge circuits are employed which capitalize on the difference in sign of temperature coefficient between a resistor printed on a substrate to that on a dielectric.

\section{ACKNOWLEDGEMENT}

We wish to acknowledge the support of this work by the South Australian Institute of Technology Research Committee and Philips Industries Holdings Ltd., Hendon, South Australia. 


\section{REFERENCES}

1. P.J. Holmes, "Changes in thick film resistor values due to substrate flexure", Microelectronics and Reliability, 12, 395-396, (1973).

2. K.E.G. Pitt, R.J. Gledhill, "Some recent advances in thick film technology", Internal Report, Middlesex Polytechnic, London, (1981). 

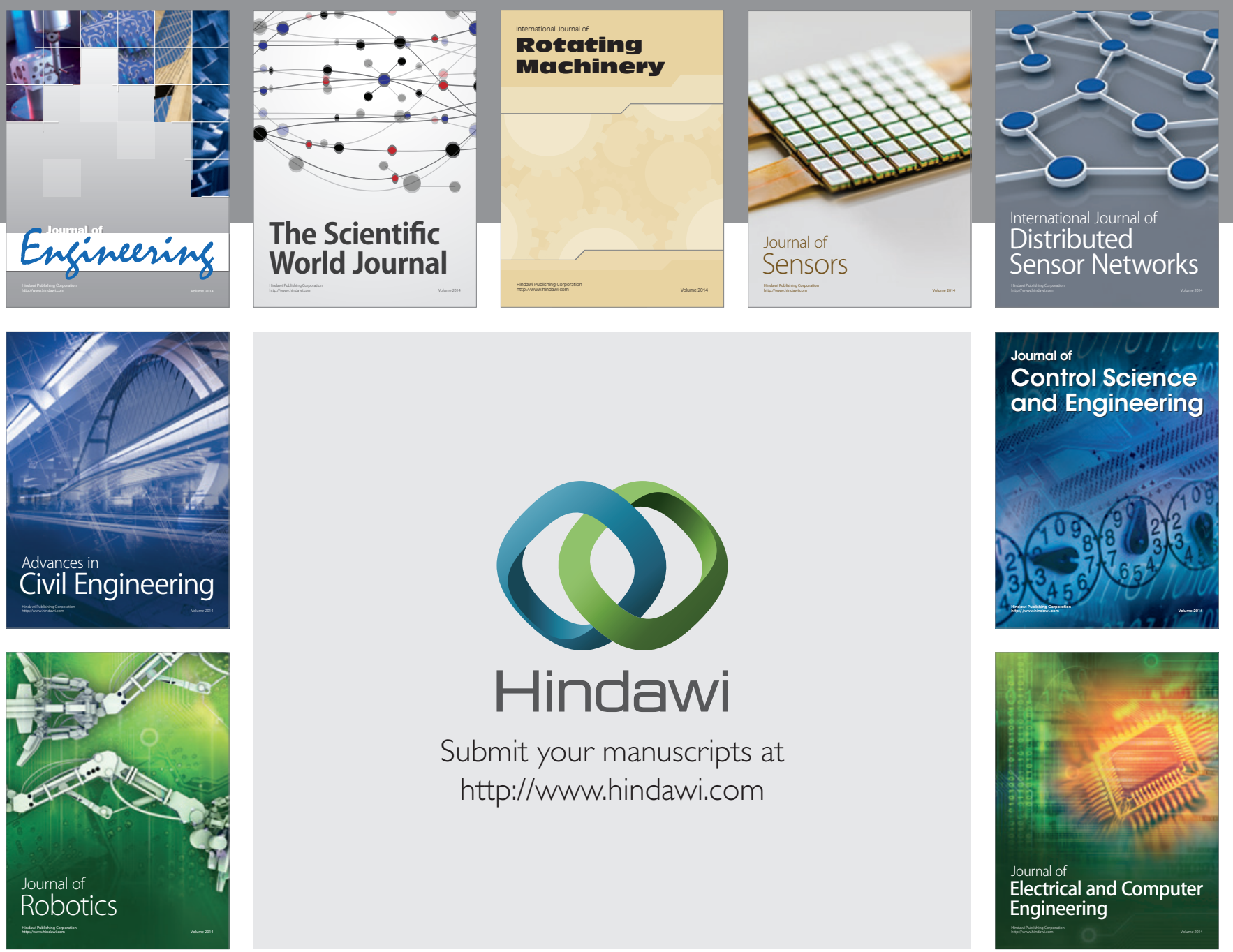

Submit your manuscripts at

http://www.hindawi.com
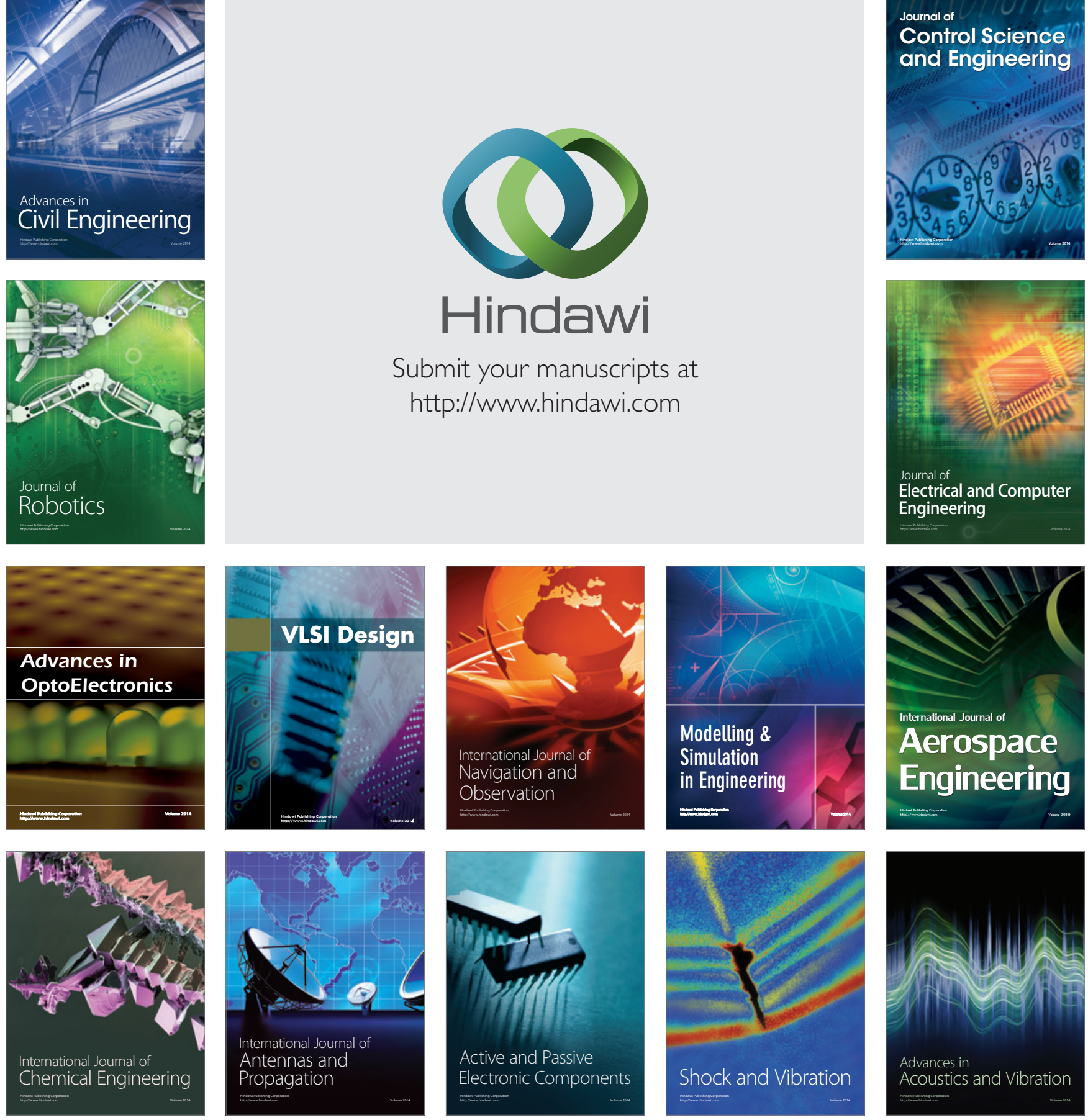